\section{SP0054 THE CONTRIBUTION OF REGULATORY B CELLS IN PROTECTING RHEUMATIC DISEASES}

C. Mauri. Medicine, University College London, London, UK

B cells are considered central to the pathogenesis of patients with rheumatic dis eases (RD) including Systemic lupus erythematosus (SLE) and rheumatoid arthritis (RA). In addition to producing autoantibodies, B cells suppressing inflammatory responses, known as regulatory $B$ cells (Bregs) are numerically and functionally defective in rheumatic diseases. The production of interleukin 10 (IL10) classically defines a Breg, however the stability and/or plasticity of this population is not well understood. In addition to producing autoantibodies, B cells suppressing inflammatory responses, known as regulatory B cells (Bregs) are numerically and functionally defective in RD patients. The production of interleukin 10 (IL-10) classically defines a Breg, however the stability and/or plasticity of this population is not well understood. Additionally, IL-10 $+\mathrm{B}$ cells have been shown to co-express pro-inflammatory cytokines such as TNFa and IL-6, further complicating Breg classification. Characterising the signals inducing Breg differentiation and the subsequent stability and/or plasticity of this population may aid in understanding the factors contributing to Breg dysfunction in RD patients. Novel findings unravelling the signals required and the stability of $B$ regs versus $B$ effector cells in RDs will be discussed.

Disclosure of Interest: None declared

DOI: 10.1136/annrheumdis-2018-eular.7731

THURSDAY, 14 JUNE 2018

\section{Sustainable healthcare in rheumatology and the role of health professionals}

\section{SP0055 HOW CAN INTEGRATED HEALTHCARE CONTRIBUTE TO SUSTAINABLE HEALTHCARE IN RHEUMATOLOGY?}

B. Vrijhoef ${ }^{1,2} .{ }^{1}$ Panaxea, Amsterdam; ${ }^{2}$ Patient and Care, Maastricht University Medical Center, Maastricht, Netherlands

Introduction: According to the World Health Organisation (WHO) "an integrated, people-centred approach is crucial to the development of health systems that can respond to emerging and varied health challenges, including urbanisation, the global tendency towards unhealthy lifestyles, ageing populations, the dual disease burden of communicable and non-communicable diseases, multi-morbidities, rising health care costs, disease outbreaks and other health-care crises". In supporting health services to become more integrated and people-centred a Framework was developed that proposes five interdependent strategies: 1. Empowering and engaging people and communities; 2. Strengthening governance and accountability; 3 . Reorienting the model of care; 4 . Coordinating services within and across sectors; 5 . Creating an enabling environment. Given that health systems are highly context-specific, the Framework does not propose a single model but interdependent (sub) strategies, policy options, and interventions that need to be adopted. What is known about the adoption of these strategies for people with rheumatic and musculoskeletal diseases?

Methods: A convenience sample of recently published reports about redesigning health care for people with rheumatic and musculoskeletal diseases is assessed against the WHO Framework on integrated-people centred health services. The five interdependent strategies, including the policy options and interventions as defined under the Framework, are assessed for presence, implementation fidelity and outcomes. Implementation fidelity refers to the degree to which an intervention is delivered as intended (i.e. in line with the Framework). Outcomes of peoplecentred care include: equity in access, quality, responsiveness and participation, efficiency, and resilience.

Results: To be presented at the conference.

Discussion: It is anticipated to find a plethora of examples of mainly single policy options and interventions, but hardly any examples of combinations of strategies. This would indicate a need for a systems approach in achieving sustainable healthcare in rheumatology.

\section{REFERENCE:}

[1] WHO. Framework on integrated, people-centred health services. Sixtyninth World Health Assembly 2016. http://apps.who.int/gb/ebwha/pdf_files/ WHA69/A69_39-en.pdf?ua=1\&ua=1 (accessed 15 March 2018).

\section{SP0056 SUSTAINABLE HEALTHCARE - IT TAKES A VILLAGE}

\section{B.A. Slusher. Physician Assistant Studies, University of Texas Medical Branch,} Galveston, USA

Healthcare delivery is at a crossroads for potential paradigm change due to budget constraints, complexity of care, increasing performance measures and implementation of electronic health records. Additionally, recent reports of high levels of stress and burnout amongst healthcare providers has been linked to decreased patient safety and medical errors. Complicating matters for rheumatology, is shrinking of the available workforce. The model of interprofessional healthcare delivery holds promise as a means to improve both patient outcomes and provider wellbeing. This presentation addresses implementation of interprofessional teams in rheumatology and provides a review of successful models and guidelines for implementation.

Disclosure of Interest: None declared

DOI: 10.1136/annrheumdis-2018-eular.7718

\section{THURSDAY, 14 JUNE 2018}

\section{SSc: From registries to trials - do we have sufficient data and the appropriate design?}

\section{SP0057 THE COURSE OF SYSTEMIC SCLEROSIS (SSC): WHAT CAN WE LEARN FROM REGISTRIES?}

M. Matucci Cerinic. Professor of Rheumatology, Director Division of Rheumatology AOUC, University of Florence, Italy, Florence, Italy

$\mathrm{SSc}$ is a heterogeneuous connective tissue disease with an unpredictable course and a high mortality and morbidity. Although the identification of patients in early stages of disease is increasingly achievable thanks to the introduction of the new $\mathrm{ACR}^{\mathrm{EULAR}}{ }^{2013}$ classification criteria, the stratification of patients according to the risk of disease progression or of severity of disease is still an unmet need. Several potential predictive factors of disease evolution have been proposed over the years: SSc specific autoantibodies related to disease complications, serum biomarkers of internal organ involvement, age at diagnosis, sex, disease subset seem to have a prognostic value but unfortuntely all these may be present in different combinations making it difficult to identify how the disease will evolve. Data from registries of different countries confirm that the clinical presentation depends on several factors. Data from EUSTAR group data base indicate that a late age at onset (>75 years) of SSc is associated with more aggressive disease as well as the male gender, that has a more severe phenotype and a worst prognosis than female, with an increased risk of occurrence of cardiovascular involvement. The analysis of geographic variations among different SSc presentations may suggest that eastern centres care for more severe SSc manifestations in Europe. Data from EUSTAR group reported also that the main causes of deaths in SSc are due to pulmonary fibrosis, pulmonary arterial hypertension (PAH) and heart involvement (mainly heart failure and arrhythmias). Independent risk factors for mortality and their seem to be proteinuria, the presence of PAH based on echocardiography, pulmonary, forced vital capacity below $80 \%$ of normal, dyspnoea above NYHA class II , reduction of dlco, patient age at onset of Raynaud's phenomenon and the modified Rodnan skin score. Interestingly, SSc patients show an increase risk of lung cancer (especially non small cell lung carcinoma) in association with interstitial lung disease and of esophageal or cardial adenocarcinoma. Data from the database of the German Network for Systemic Scleroderma support the concept that SSc-overlap syndromes should be regarded as a separate SSc subset distinct from IcSSc and dcSSc, due to a different progression of the disease, that had an intermediate rate of disease progression in between IcSSc and dcSSc, different proportional distribution of specific autoantibodies, and of different organ involvement. Therefore, the use of registries is today of paramount importance to obtain significant data about several unmet needs in SSc.

Disclosure of Interest: None declared

DOI: 10.1136/annrheumdis-2018-eular.7871 